\title{
Publicaciones periódicas y vida intelectual en Nicaragua: El Mentor nicaragüense de Frutos Chamorro (1841-1842)
}

Miguel Ayerdis ayermi2003@yahoo.com

Recepción: 21-04-16 /Aceptación: 18-07-2016

\section{Resumen}

En este ensayo se hace una aproximación a la vida cultural nicaragüense de la primera mitad del siglo XIX, en diálogo con E.B. Burns quien en Patriarcas y pueblos. El surgimiento de Nicaragua. 1798-1858, afirma que en la Nicaragua de esos primeros años de vida republicana, habia una actividad intelectual digna de documentar. Sobre este aspecto, se estudia a uno de los personajes controversiales en la historia de este país, Fruto Chamorro, a partir de su veta intelectual, principalmente en su faceta de periodista, al frente de El Mentor Nicaragüense (1841-1842). Otro aspecto que se integra al tema, es la labor de documentalista de Orlando Cuadra Downing, quien desde la Revista Conservadora del Pensamiento Centroamericano, divulgó documentos valiosos para la historia de Nicaragua. Por ello, este trabajo además de problematizar acerca de la cultura de Nicaragua en las primeras décadas del siglo XIX, hace una valoración al trabajo documental.

Palabras claves: cultura nicaragüense, vida intelectual, publicaciones periódicas, gestión documental

\begin{abstract}
.
This essay is an approximation of cultural Nicaraguan life during the first half XIX century in dialogue with E.B. Burns who in "Patriarcas y pueblos. El surgimiento de Nicaragua. 1798-1858", who says that in the first period of the republican life of Nicaragua, there was such intellectual activity that merit to be documented. This paper study one of the most controversial personage in this country: Fruto Chamorro. Beginning from his intellectual background, mainly as a journalist leading El Mentor Nicaragüense newspaper (1841-1842). The documentation work done by Orlando Cuadra Downing through the Conservadora del Pensamiento Centroamericano magazine, where released valuable documents for Nicaraguan history is also addressed. Because of that this paper in addition to problematize about Nicaraguan culture at the beginning of XIX century, does an assessment of documentary work.
\end{abstract}

Keywords: Nicaraguan culture, intellectual life, documentary management, newspaper publication. 


\section{Introducción}

Hablar de intelectuales en Nicaragua durante la primera mitad del siglo XIX pareciera anacrónico, sabiendo el lastre del período, y la experiencia --opuesta a la anterior--, de la segunda mitad de ese siglo, donde con propiedad se puede afirmar que se configura un grupo de personas, con formación académica, que ejercen en su cotidianidad, una profesión u oficio, siendo uno de ellos el de la crítica (generadora de opinión pública) sobre aspectos sensibles de la vida política, económica y cultural de la época. En lo referente al "período de anarquía" (primera mitad del siglo XIX), --cognomento dado por la historiografía tradicional nicaragüense al período que va de 1824 a 1858, E. Bradford Burns (1998) afirma de la existencia de "cierto desarrollo intelectual" (p.41). Este criterio, poco extendido en los estudios sobre el siglo XIX en Nicaragua, requiere de mayores reflexiones, $y$ este ensayo es un aporte al mismo.

La afirmación señalada de Burns, hace que se reflexiones sobre los textos del siglo XIX escritos por intelectuales de esa época, recogidos en la sección La Voz Sostenida, de la Revista Conservadora (nombre de su primera etapa) y Revista Conservadora del Pensamiento Centroamericano (segunda etapa) por Orlando Cuadra Downing, durante la década de los 60 del siglo pasado. En el prólogo para la edición en forma de libro del primer tomo (publicado hasta el año 2007), el compilador, Cuadra Downing, enfatiza el carácter de "antología del pensamiento nicaragüense" por medio de cual se puede hacer una relación del pensamiento libertario y la "formación de la nacionalidad patria” (2007, p.34). Más adelante, señala el antologista que la producción escrita (literaria le llama) en temas filosóficos o doctrina- rios son escasos, aspecto sobre los cuales se hará referencia en este ensayo (2007; p.35).

El propósito de este trabajo es aproximarse a la vida cultural nicaragüense de la primera mitad del siglo XIX con el fin de problematizar acerca de la afirmación de Burns, de la existencia de una actividad intelectual en Nicaragua en estos primeros años de vida republicana. Otro propósito, consiste en la identificación de los intelectuales y su producción escrita, partiendo de la selección que hiciera Cuadra Downing y los espacios de divulgación y circulación de los textos. Por último, se hará mención del caso de un "intelectual del siglo XIX" que combinó la pluma y la acción política con éxito, dejando un legado, aún no estudiado a profundidad, y que marcó la historia del país: Fruto Chamorro (1804-1855) y el periódico El Mentor Nicaragüense (1841-1842)

\section{Contexto del estudio}

Para iniciar, es importante hacerlo comentando estudios sobre la vida cultural en el siglo XIX en Nicaragua (Quizás sea válida para toda Centroamérica). Algunos hacen referencia a un despertar intelectual después de la primera mitad de este siglo, de manera particular a partir de lo que la historiografía oficial nicaragüense (tradicional) ha definido como período de los "Treinta años conservadores" (véase a Cuadra Pasos, C.; Teplitz, B.; Urbina, N.; Cuadra Downing, O.; Cruz S. A.; Ayerdis, M. ;).

Si bien, la mayoría de estos estudios, hacen alusión a figuras notables dentro de la cultura y la política (Gregorio Juárez, Jesús de la Rocha, Enrique Guzmán, Jerónimo Pérez, Tomás Ayón, Anselmo H. Rivas, José Dolores Gámez), su vida intelec- 
tual y los ambientes en que se movieron, apenas se dibujan en los escritos de la época, dejando la duda de si en verdad existió una ambiente de "vida intelectual" como tal en Nicaragua, durante la primera mitad del siglo XIX.

De lo que sí hay consenso entre los especialista de Nicaragua en esta época, es la relación existente entre "vida intelectual" (ambiente intelectual) y publicaciones periódicas (Ayerdis, Herrera C., M. Á.). Los periódicos y revistas, representan espacios simbólicos de encuentro y debate de ideas (siguiendo a Chartier), donde los deseos y realidades alrededor del modelo de estado y de nación a construir y los problemas (institucionalidad, educación, ciudadanía, soberanía, progreso) que enfrentan para alcanzar esa metas, se hacen sentir con fuerza, entre la élite dominante, heredera de los poderes políticos y culturales del Antiguo Ré34 gimen y los abanderados de las nuevas corrientes de pensamiento.

La periodización (dos grandes períodos) elaborada por la historiografía tradicional que da cuenta de lo ocurrido en el siglo XIX, contraponiendo, un período de anarquía y una de paz, está mediada por dos factores: la primera, es una suerte de "reduccionismo intelectual", salida de una documentación del período. Sin restarle méritos a estudios que relacionan la inserción de los países de Centroamérica al mercado mundial, con los avances en vida material de los habitantes de estos país, no se puede ser tan contundente y señalar que la vida intelectual y cultural de la segunda mitad del siglo XIX, en el caso de Nicaragua (para hablar de nuestro objeto de estudio), era dinámica y de gran riqueza, a lo sumo habían --como lo sugieren esos mismos autores (Sergio Ramírez, Arturo Cruz Sequeira, Benjamín Teplitz, Charles Stan- sifer, Miguel Ayerdis) manifestaciones incipientes de vida cultural, en la capital y algunas ciudades de importancia.

La otra, es la creencia del período de la "Anarquía" (dentro de una concepción que parte de la oposición, liberales-conservadores) como una suerte de Edad Media europea (tal como nos lo enseñaron en las escuelas muchos años atrás), de un período oscuro, tenebroso, donde nada se puede rescatar. El Estudio de Frances Kinloch Tijerino (1999) es uno de los pocos que rebate esa concepción al problematizar sobre la identidad y la construcción política y cultural del estado en este período nicaragüense (1821-1856). Otro aspecto que resalta el trabajo de Kinloch Tijerino y el de Herrera C. (1999) también, es la comprobación de las posibilidades de hacer estudios de la primera mitad del siglo XIX, sabiendo usar con creatividad y racionalidad, las fuentes dispersas relacionadas con Nicaragua de este período.

El énfasis puesto de manera sistemática por la historiografía nicaragüense, a este período, es de inestabilidad política. Con ello se busca sustentar los argumentos más usados por los intelectuales de esta época, quienes esgrimieron como causa principal de los problemas del país, las asonadas o levantamientos armados, producto de las divisiones localistas entre las élites que buscaban el control del estado. Ahora bien, el proceso de rescate o visibilización de figuras que podrían considerase dentro de la categoría de intelectuales, debe tomar en cuenta las condiciones históricas de la época, donde el fuerte componente localista, hace ver un pensamiento atomizado, donde.--por ejemplo-- el concepto de "patria" utilizado en el discurso público, es tan ambiguo, que no se sabe si se habla de los nuevos Estado/nación surgidos 
de la disolución de la Federación (1838), las ciudades donde viven las élites, o lo que comprendía el Reino de Guatemala (Kinloch Tijerino, 1999).

\section{“Vida intelectual” y publicaciones periódicas}

La identificación del clima intelectual en las sociedades del siglo XIX centroamericano y nicaragüense, debe de pasar por el conocimiento y/o comprensión del funcionamiento de las publicaciones periódicas producidas en cada uno de estos países. La experiencia del Editor Constitucional en Guatemala (1808-1926), es un claro ejemplo de la forma en que los intelectuales de la época creaban estos espacios (físico y simbólicos) para debatir acerca de los diversos temas, teóricos (doctrinarios), políticos o económicos.

En el caso de Nicaragua, la situación no es distinta al del resto de países de Centroamérica. Las publicaciones periódicas, surgidas después de la llegada de la imprenta en 1830, son los puntos de inicio para hacer un recuento de la vida intelectual y explicar las preocupaciones que estos tenían en relación a la nación, "lo nacional", a como lo sugiere Benedict Anderson (1993, Anderson; 2005; Ayerdis). En el trabajo citado de Bradford Burns (1998), se menciona a los periódicos como los espacios naturales donde se debatía sobre diversos temas de la vida nacional. No viene al caso, ni es el propósito hacer una historia de los impresos o publicaciones periódicas, sino destacar a algunos personajes, que de alguna manera, aparecen como figuras públicas en esos períodos iniciales de la construcción del estado nación y que con sus escritos publicados en estos espacios, representan la base "doctrinaria" o "filosófica" que Cuadra Downing no pudo encontrar, cuando hizo la revisión documental del siglo XIX.
Otro aspecto sobre los intelectuales es lo que señala Burns para esta época en América Latina: además de periodista eran "activistas políticos y sociales" (p.41). Es bueno recordar que una de las formas de la canalización de las ideas y la socialización de las demandas de las elites de esta épo$\mathrm{ca}$, era por medio de las universidades existentes (Guatemala y León) y los espacios públicos donde la elite letrada se daban cita y uno de estos eran las publicaciones periódicas u hojas sueltas.

Esos espacios públicos modernos eran las tertulias, las cuales proliferan poco antes de la independencia y continúan con fuerza en la etapa de construcción de la república, en el siglo XIX. Como bien lo dice Renán Silva (1998), las tertulias, "son espacios privados o semi privados, o aún espacios públicos, utilizados de manera privada, de conversaciones y discusiones que funcionaban en las habitaciones de estudiantes" (p. 81-82). Las Sociedad Económica de Amigos del País y la Gazzeta de Guatemala (1797) es una publicación, creada como plataforma o tribuna de expresión por prominentes intelectuales independentistas, con la finalidad de socializar sus puntos de vista políticos y económicos con el público letrado (Bonilla, A. p.121).

La relación tertulia o clubes y publicaciones periódicas, representa la confluencia de dos espacios (el real y el simbólico) cultural letrado o intelectual de gran valor.--por lo que representa como fuente-- para la comprensión de la sociedad del siglo XIX. La mayor parte de los periódicos aparecidos desde 1830 en Nicaragua, tienen como finalidad la divulgación de ideas políticas en el marco de contiendas electorales o de crisis políticas. Durante el siglo XIX era habitual que cada club o tertulia política que apoyaba un bando o facción 
política, editara proclamas, octavillas o periódicos (Kinloch, Tijerino. 1999: p. 122-127).

El primer periódico publicado en Nicaragua, El Telégrafo (1835), en una de las páginas interiores de su primer número señalaba:

En los tiempos de anarquía, cuando se horroriza el género humano al contemplar los bárbaros desórdenes que se comenten y que pasman a la admiración misma, la libertad e igualdad, se oye en boca de todos. E incluso en que con más fiereza la atacan y destruyen con sus obras. Los sans-culottes de Francia, los gibelinos de Florencia y todos los que han querido contrariar las instituciones más benéficas, se han valido de esta voz encantadora... [el periódico]" (20 de agosto de 1835)

36 Estas ideas de gran significación, por el contexto de inestabilidad que evoca, eran leídas por las elites afines al bando que defendían, dado que en estas iniciales publicación periódicas, estaba ausente la cultura de la réplica en el mismo periódico. Eran periódicos para ser leídos por los seguidores letrados. Otra experiencia de estos espacios públicos simbólicos, es la del periódico Mentor Nicaragüense (1941-1942), fundado y editado por Fruto Chamorro, miembro de la élite política de Granada, quien hace uso de este recurso comunicacional, con el fin de hacer sentir su liderazgo por medio de los escritos que publica.

\section{El periódico el Mentor y Fruto Chamorro}

En el periódico El Mentor está recogido todo el ideario político de Fruto Chamorro (1804.1855), considerado el fundador del Partido Conservador de Nicaragua. El lema que acompañaba el título del periódico: "no hemos nacido sólo para nosotros, sino también para la Patria" (CD. Periódicos de Nicaragua del siglo XIX. V.2; Catálogo Periódicos, TI, 1992), refleja la voluntad política e intelectual de esta figura nacional, quien habiendo nacido y vivido su juventud, Guatemala, influyó con su pensamiento y acción, la política del país hasta principios del siglo XX.

El Mentor, al igual que la mayoría de los periódicos de esta época, busca llenar vacíos que el Estado por su debilidad institucional y carencia de recursos no podía asumirlos, como era la educación. En este sentido, la educación era concebida bajo un criterio racionalista ilustrado donde se debía de educar al pueblo sobre los deberes públicos, moral y de justicia. El primer número del mentor trae un artículo donde establece los propósitos de la publicación:

Procurar la ilustración de los pueblos, presentándoles doctrinas claras y sencillas de las materias que más les interesan: manifestarles todo aquello que influya en su bien y prosperidad: formar el espíritu público sobre las sólidas y verdaderas bases en que se funda nuestro sistema de gobierno; y poner al alcance de todos cuantas ideas y pensamientos hagan relación con aquellos objetos (Chamorro Zelaya, 1960; p. 27).

Es importante destacar que la mayor parte de los artículos que aparecen en El Mentor son escritos por Fruto Chamorro, quien demuestra, con la variedad de temas abordados y la información que despliega, una sólida formación académica, la cual adquirió en la Universidad de San Carlos de Guatemala, donde se graduó de bachiller en 
Filosofía y sobresalió en matemáticas (Chamorro Zelaya, p.11).

La historia de este personaje en la vida política nicaragüense es singular. Era hijo natural de Pedro José Chamorro Argüello, un potentado vecino de Granada, y de reciente migración a Nicaragua y de Josefa Pérez, guatemalteca de la que se conoce poco de su vida. Toda su infancia y adolescencia la pasó en Guatemala, al lado de la familia materna (desde niño llevaba el apellido de la madre). Según cuenta uno de sus biógrafos, Pedro Joaquín Chamorro Zelaya, la llegada de Fruto a Nicaragua obedeció a una solicitud hecha por la viuda de Pedro José Chamorro, doña Josefa Alfaro y Monterrroso, quien a petición de su esposo, poco antes de morir en 1824, le llamó y convenció para hacerse cargo de los negocios de su difunto padre, en su condición de hijo mayor. El 27 de octubre de 1829 doña Josefa Alfaro hizo el traspaso a Fruto Chamorro (quien ya aparece con el apellido de su padre) del poder generalísimo de los bienes de la familia ante los oficios notariales de don Dionisio de la Quadra (Chamorro Zelaya, pp.).

A los pocos años de establecido en Granada, en su calidad de jefe del clan Chamorro, es encarcelado por haber participado en las luchas de facciones (Chamorro Zelaya. p.21). En el año de 1836, es elegido diputado de la Asamblea ordinaria del Estado, donde aboga por la creación de instituciones educativas, logrando pocos años después, la fundación de la Universidad de Oriente y Mediodía, desde donde daría a conocer su pensamiento político, fundando el periódico El Mentor Nicaragüense (Chamorro Z. p. 23).

Su ideario político se sustentaba en una sólida formación libresca (lectura de clásicos europeos), y en la experiencia vivida en Guatemala y Nicaragua, identificando como causa fundamental de la inestabilidad política en ambos estados, la debilidad del poder ejecutivo (Chamorro Z. p. 23). La idea del orden, libertad, autoridad y legitimidad, serán los ejes del pensamiento que lo acompañarán hasta su prematura muerte en 1855.

Para los comicios de representantes y de autoridades municipales de 1841 exhortaba, desde las páginas de El Mentor Nicaragüense, a los electores a participar de manera consientes, sabiendo que: "Los actos electorales deben ser absolutamente libres. Todo lo que tienda a impedir la libertad es un atentado. Por esto, es prohibido, aún a la autoridad, tener en ellos más intervención que la necesaria para conservar el orden" (Chamorro Zelaya, p. 31).

Se le considera el padre del Partido Conservador porque defendió ideas moderadas, sustentada en una moral cristiana, que prescribía que el hombre debe actuar con cordura y haciendo el bien. En el Mentor del 4 de diciembre de 1841, en ocasión de las elecciones de autoridades,--ya mencionada--, señalaba:

Estos actos (el proceso electoral) son majestuosos y como tales deben de estar acompañados, no de una libertad tumultuosa, insolente y desenfrenada, sino de una libertad moderada, racional, comedida, de libertad propia de patriotas libres e ilustrados, y no de una libertad que degenere en licencia o libertinaje (p.1).

En el Mentor del 6 de noviembre de 1841 establecía sus ideas morales, sustentada en concepciones cristianas pero también lógicas (racionales). Si bien su premisa moral parte de la idea "Amar a 
Dios sobre todas las cosas y al prójimo como a uno mismo", en el sustrato subyace la idea de la necesidad de velar por la comunidad por la colectividad, a partir del respeto a las diferencias y a la libertad:

Todos debemos de empeñarnos en difundir las máximas de amor al prójimo, de no querer para otros lo que no queremos para nosotros y de hacer a los demás el bien que quisiéramos se nos hiciese: todos debemos de inculcarlas, y predicarlas tanto con las palabras como con el ejemplo (P.1)

Pero donde se observa una posición que fundamentó el catalogarlo Conservador de pensamiento (y desde el punto de vista político), es en relación a la instrucción (educación), donde manifiesta que debe ser diferenciada para las diversas clases sociales:

...es preciso también advertir, con el Señor Filangieri, que popularizar la instrucción, no es darla con uniformidad a todos los individuos de la sociedad, porque como dice este acreditado autor: ella exige, --hablando de educación--, que se dé a cada uno según sus circunstancias y el objeto a que está destinada. Que el colono reciba la instrucción necesaria para ser ciudadano y colono, y no para ser Magistrado o caudillo... (El Mentor, 6 de noviembre 1841 ; p. 67)

Otro aspecto de las ideas que deben de rescatarse del pensamiento de Chamorro, es su creencia en el establecimiento de una entidad superior que aglutinara y resguardara la soberanía de los recién creados estados independientes, después de la ruptura del pacto federal en 1838. Siendo una figura pública reconocida en Nicaragua, el pe- riodista Chamorro, se hace eco de la alarma que causara entre la clase política centroamericana, las acciones inglesas en San Juan del Norte, en costa Caribe nicaragüense, y la Unión en El Salvador, donde el Superintendente inglés, Chatfild con arrogancia y fuerza presionaba a los gobiernos de Centroamérica para que "honren las deudas contraídas por la extinta Federación" (Kinloch Tijerino; p. 74-81; Chamorro Zelaya, p.27-40).

En la edición del 4 de diciembre de 1941, de El Mentor, aparece una nota, sin duda redactada por Fruto Chamorro, donde hace un llamado a los políticos a la unidad:

...el asunto de San Juan es muy delicado. Su decisión debe ser menos obra de la fuerza que de medidas políticas y diplomáticas que no pueden adoptarse con seguridad si siguen los Estados separados unos de otros, como hasta ahora, sin tener unidad nacional y que para conseguir esto, todos debemos agotar los arbitrios, porque de otra suerte, peligra la independencia, no sólo de uno, sino de todos ellos...

La creación de la efímera Confederación Centroamérica, conformada por Honduras, El Salvador y Nicaragua, surgido del Pacto de Chinandega de julio de 1842, --pese a las diferencias y contradicciones entre las elites de estos países--, se debió en parte, a la campaña emprendida en el Mentor Nicaragüense por Fruto Chamorro. El primer Supremo delegado de esta Confederación, cuya sede se designó San Vicente en El Salvador, fue ocupado por Fruto Chamorro. 


\section{La Voz Sostenida y el pensamiento nicaragüense del siglo XIX}

La selección de textos dados a conocer en la Revista Conservadora (1960-64) y Revista Conservadora del Pensamiento Centroamericano (1964-1994) en la década del 60 del siglo XX por Orlando Cuadra Downing, representa una de las preocupaciones más sentidas de los intelectuales nicaragüenses de los últimos tiempos, por "documentar el origen de la nación nicaragüense". Desde Jerónimo Pérez (1828-1884) hasta Sergio Ramírez, novelista, pasando por José Coronel Urtecho (1906-1994) y Pablo Antonio Cuadra (1912-2001) poetas, la construcción de un relato que narre los orígenes de la nación nicaragüense, en sus detalles y coherencia, ha chocado con la problemática de carencias o dispersión de fuentes históricas.

Pero más que las carencias documentales, está la falta de consenso en la forma en que debe de construirse el relato que explique, desde el punto de vista histórico, esa fundación de la nación y los pormenores de su evolución. Los textos de historia escritos por Tomás Ayón (1820-1887) y José Dolores Gámez (1851-1918), considerados como referencias de los primeros intentos por explicar, lo que Nicasio Urbina llama "la fundación de la nacionalidad nicaragüense", y las implicaciones que este enunciado contiene, en términos de pertenencia e identificación con una colectividad "imaginada mayor" (siguiendo a Anderson), han sido más bien, un lastre en términos explicativos de los episodios más sensibles del siglo XIX. El primero (Ayón), apenas describió el proceso de independencia (el título del libro señala hasta 1852; incorpora un apéndice -texto breve-- acerca de acontecimientos de 1821-1824) y el segundo (Gá- mez), más completo en términos cronológicos de la historia nacional, le dio un sesgo de facción, donde los liberales tenían mayor protagonismo.

El sesgo de facción (ideológico hasta cierto punto) implícito en el texto de Historia de Nicaragua de Gámez, publicado a finales del siglo XIX (1896), tuvo su antecedente en otros textos de gran importancia como referencia documental: los escritos de Jerónimo Pérez (1828-1884). Este intelectual de la segunda mitad del siglo XIX, testigo y participante de la guerra civil y la guerra filibustera de 1854-1857, escribió una serie de textos, la mayoría de ellos publicados en el periódico que él fundara de nombre La Tertulia (1876-1877). Pérez, perteneció al bando conservador, razón por la cual sus puntos de vista, serán más favorables para su causa que para la de los liberales.

La falta de consenso de un relato explicativo de los acontecimientos políticos de la primera mitad del siglo XIX y la Guerra Nacional, ha generado un "vacío histórico", en las generaciones subsiguiente de nicaragüenses, lo cual explica en parte, los esfuerzos de Coronel Urtecho y su libro Reflexiones sobre la historia de Nicaragua (2001), publicado en tres tomos en la década del 60 del siglo pasado, y la Antología (La Voz Sostenida) publicada por entregas, por Orlando Cuadra Downing.

En Reflexiones sobre la historia de Nicaragua, Coronel Urtecho, retrata el quehacer del intelectual del siglo XIX, desde los textos de historia. Señala que en la historia escrita se repite "la historia vivida como guerra civil": "Esta se ha escrito desde la guerra misma y como parte de ella. Los libros y folletos de los historiadores, puede decirse que en cierto modo, correspondían a los cañones y fusiles de los combatientes" (p.5). Más 
adelante señala: "Hay por lo menos dos historias distintas, aunque complementarias de Nicaragua: la liberal y la conservadora" (p.5).

La Voz Sostenida es el nombre de la sección especial de la Revista Conservadora/Revista Conservadora del Pensamiento Centroamericano, donde Orlando Cuadra Downing, en su calidad de redactor principal, publicaba documentos históricos, de lo que llamaba "el pensamiento nicaragüense". Esta idea de rescate y memoria, formaba parte del esfuerzo que el fundador y director de la revista, Joaquín Zavala Urtecho (1891-1971) realizaba como legado a las generaciones futuras, de los avatares que enfrentaba el país en esa época: "daremos acogida en nuestras páginas -señalaba en el primer número de la revista - a todo lo que despierte memoria de nuestro pueblo (...) deliberadamente conducido a olvidarse de lo propio por 40 la superficial educación que se imparte". (RCPC, pág. 3)

En esta sección se recogen textos considerados emblemáticos para la comprensión de la historia de Nicaragua, de manera particular en el siglo XIX. En relación a los textos y autores de la primera mitad del siglo XIX, publicados en la Voz Sostenida, se recogen trece. Los autores son conocidos por sus diversas facetas que presentaron en su vida pública (político, abogado, religioso, periodista, cientísta), entre otros: José Antonio López de la Plata (Tomás Ruíz (1777-¿’?, Manuel Antonio de la Cerda (1780-1828), Juan Argüello, Desiderio de la Quadra (1779-1849), Miguel Larreynaga (1772-1847), Toribio Tijerino Pomar (1808-1850), Rosalío Cortés, Sebastían Salinas, Gregorio Juárez (1800-1879) y Fruto Chamorro.
El hilo temático de los textos alusivos a temas políticos y culturales, de estos autores, tienen que ver con preocupaciones sentidas en la época, como era la libertad, el progreso, la paz y la justicia, y "la construcción de la patria", ¿cuál y qué modelo seguir? Era la pregunta que introduce el discurso de Don Manuel Barberena en 1849 en ocasión del XX del XXVIII aniversario de la independencia y contestaba el "Republicano": "[ser republicano], es vivir bajo el gobierno más natural del hombre, porque es el gobierno de la razón. Es la forma en que el espíritu halla libres todos sus resortes para hacerse y hacer todo el bien posible..." (2007, Cuadra Downing, p.228).

El compilador Cuadra Downing se preocupó por reunir textos que dialogaran en contextos específicos de la vida nacional con el fin de reconstruir espacios de debate político, como el generado entre don Rosalío Cortés y José María Estrada (¿?1856), en relación al "gobierno fuerte" impuesto a expensa del orden constitucional, por Fruto Chamorro en 1853. En ese debate público, el Doctor Cortés replica el escrito de Estrada quien defiende la ruptura institucional y la imposición de un régimen de los ricos:

Compañero: usted y yo estamos animados de iguales propósitos en pro de la patria nicaragüense y de la culta Granada; sólo diferimos en los medios de labrar su dicha y de hacerla grande, feliz y poderosa. Pero no debemos separarnos del sistema republicano, verdaderamente liberal, que es el que se propusieron los mayores al emanciparse del gobierno monárquico de España: los pueblos han saboreado este bello sistema y si nosotros se lo cambiamos por esa oligarquía, provocaremos una revolución de 
resultados incalculables, en la cual nuestra querida Granada puede quedar en escombros... (2007, 165-166)

Una de las dificultades para la ubicación de la mayor parte de estos autores (a excepción de Larreynaga y Fruto Chamorro), y sus escritos en un contexto sociocultural determinado de principios del siglo XIX, es -además de la sistematicidad de sus publicaciones-- la escasa referencia hasta ahora encontrada, de sus actividades. La producción intelectual se presenta limitada, comprende algunas cartas, discursos, escritos didácticos y proclamas dispersas. No obstante, es un buen punto de partida para hacer comparaciones o relaciones con otros autores y contextos con el fin de tener un corpus explicativo aceptable de los temas de la época que se quieren trabajar, en este caso, el de la vida intelectual.

Es importante señalar que las publicaciones periódicas existentes durante la primera mitad del siglo XIX en Nicaragua, --pese a lo incompleto de las colecciones-reflejan y/o reproducen el ambiente y/o clima social y político que se vivía en un contexto tan complejo como el de esta época. De igual manera, por el tipo de empresa periodística, incipiente, los que escribían la mayor parte de los textos eran los redactores. Por ello, esta veta de rescate del pensamiento de algunos intelectuales, como Fruto Chamorro, Gregorio Juárez, entre otros, no importando la dispersión y fragmentación de las publicaciones periódicas, representa una labor todavía pendiente en algunos casos, como el de Nicaragua.

Un ejemplo de lo anterior, es la vida del sabio Gregorio Juárez. Este personaje, nacido en 1800, formado académicamente en Nicaragua y Guate- mala, no se conoce mucho de vida intelectual, en sus primeros 50 años. Una manera de rescatar su pensamiento es por medio de los escritos en los periódicos que él fundó y dirigió, sabiendo que el Redactor/Director era el que escribía, si no todas, la mayoría de las notas que se publicaban.

En el periódico Telégrafo Nicaragüense, fundado y dirigido por el sabio Juárez en la ciudad de León en 1835), se aprecia el sentimiento de alegría de estos pueblos por haber acabado con la violencia. Sin duda que es el punto de vista del autor (Juárez):

Es halagüeña la perspectiva del Estado de Nicaragua, tranquilo. Tres lustros han transcurrido desde la regeneración política de Centroamérica y todos habían sido marcados infaustamente con los desagradables nombres dados a las guerras civiles se han sucedido. Afortunadamente se ha dejado ver la aurora del cuarto lustro con sus refulgentes rayos de hermosa luz simbolizando la paz, el orden, la regla pública, la prosperidad, y la mejor organización social. $\left(\mathrm{N}^{\circ} 1\right.$ 20 de agosto, 1935)

\section{Conclusiones}

La vida intelectual en la Nicaragua del siglo XIX debe de reconstruirse en torno a las publicaciones periódicas y de las tertulias o clubes políticos aparecidos a lo largo de este siglo. Figuras destacadas en la vida política y cultural, supieron utilizar estos espacios públicos de difusión para dar a conocer su pensamiento y participar de los debates o "luchas" públicas relacionados a los temas y problemas vividos por los nicaragüenses (al menos los sectores urbanos) de la época; las contradicciones 
entre las élites políticas, y los conflictos con los países vecinos y las potencias.

La carencia de un sistema educativo estatal y de políticas culturales que fomentara el intercambio de ideas es una realidad inobjetable en la Nicaragua de la primera mitad del siglo XIX. Las universidades de León y la efímera universidad de Oriente y Mediodía (Granada) fueron dos instancias -junto con la de Guatemala-que contribuyeron a la promoción de intelectuales que luego incursionarían en la política y el cultivo de las letras y las ciencias. De igual manera, se puede decir de las páginas de las publicaciones periódicas, las hojas sueltas que proliferaban en forma de octavillas o en escritos dispersos con fines de publicidad o arengas políticas pero también por medio de prácticas orales (2001, Herrera M.A.)

El pensamiento de muchos intelectuales nicaragüense de la primera mitad del siglo XIX es aún desconocido, no tanto por la carencia de fuentes, sino por la poca dedicación al rescate a partir de proyectos de mediano y largo alcance, de aquellos escritos publicados en los periódicos de la época. Es verdad que las colecciones de estos periódicos están incompletas y en el peor de los casos han desaparecidos, pero también lo es, que existen ejemplares dispersos en bibliotecas y centros de documentación en la región latinoamericana o fuera de ellas que esperan ser recopiladas y sometidas a un estudio crítico.

\section{Referencias}

-Anderson, B. (1993) Comunidades imaginadas. Reflexiones sobre el origen del nacionalismo. México: Fondo de Cultura Económica.

-Ayerdis, M. (2005) Publicaciones periódicas, formas de sociabilidad y procesos culturales en Nicaragua (1884-1926). Managua: Banco Central de Nicaragua.

Ayón, T. (1993) Historia de Nicaragua: desde los tiempos más remotos hasta el año de 1852. Managua: Fondo de Promoción Cultural-BANIC.

-Bonilla Bonilla, A. (1999) Ideas económicas en la Centroamérica ilustrada. 1793-1838. El Salvador: FLACSO

-Burns, E.B. (1998) Patriarcas y pueblos. El surgimiento de Nicaragua. 1798-1858. Taller de Historia. Cuaderno No5. Managua: Instituto de Historia de Nicaragua y Centroamérica.

-Cuadra Downing, O. (2007) La voz sostenida. Antología del pensamiento nicaragüense. Managua: PAVSA.

-Cruz Sequeira. A. (2003) La República Conservadora de Nicaragua. 1858-1893. Managua: Fundación Vida.

-Cue. A. (1999) Cultura escrita, literatura e historia. Conversaciones con Roger Chartier. México: Fondo de Cultura Económica.

-Chamorro Zelaya, P.J. (1960) Fruto Chamorro. Managua: s.n. 
-Gámez, J. D. (1975) Historia de Nicaragua. Managua: Fondo de promoción cultural del Banco de América.

-Herrera C. M. A. (1999) Bongo, bogas, vapores y marinos: historia de los "marineros” en el Río San Juan. 1849-1955. Managua: Centro Nicaragüense de Escritores.

(2001) Un nuevo espacio político: la prensa escrita. Nicaragua 1830-1867. En: IPGH Memorias del IV Simposio Panamericano de Historia. San José, Costa Rica: IPGH.

-Instituto de Historia de Nicaragua y Centroamérica (2002) Periódicos de Nicaragua del siglo XIX. (2da edic. V I y II) [CD-ROM]. Managua: Instituto de Historia de Nicaragua y Centroamérica.

-Instituto Nicaragüense de Cultura (1992). Catálogos de periódicos y revista de Nicaragua (1830-1930. TI. Managua: Instituto Nicaragüense de Cultura.

-Kinloch Tijerino, F. (1999) Nicaragua: Identidad y cultura política (1821-1858). Managua: Banco Central de Nicaragua.

-Ministerio de Educación Pública (1954) Escritos del Doctor Pedro Molina. El Editor Constitucional. 2T. Guatemala: Editorial del Ministerio de Educación Pública.
-Pineda de Mont, M. (Comp.) (1999) Biografía y otros documentos originales relativos a Miguel Larreynaga. Managua: Programa textos escolares nacionales.

Ramírez, S. (1983) Balcanes y Volcanes. Managua: Editorial Nueva Nicaragua.

-Silva, R. (1998) Prácticas de lectura, ámbitos privados y formación de un espacio público moderno. Nueva Granada a finales del Antiguo Régimen. En: Guerra, F.X. y Lempérière, A. et.al. Los espacios públicos en Iberoamérica. Ambigüedades y problemas. Siglos XVIII-XIX. México: Centro Francés de Estudios Mexicanos y Centroamericanos/Fondo de Cultura Económica.

-Teplitz, B. (1988) El orden constitucional del régimen de Zelaya. En: Carmen C. (Comp.) Textos de la historia de Centroamérica y el Caribe: Nicaragua. México: Instituto Mora.

-Urbina Guerrero, N. (2007) ¿Cómo las voces sostienen las acciones?: textos fundacionales de la nacionalidad nicaragüense. En: Cuadra Downing,O. La voz sostenida. Antología del pensamiento nicaragüense. Managua: PAVSA.

-Urtecho, J.C. (2001) Reflexiones sobre la historia de Nicaragua: de la colonia a la independencia. Managua: Fundación Vida. 